\title{
The cyclin-dependent kinase inhibitor SNS-032 induces apoptosis in breast cancer cells via depletion of Mcl-1 and X-linked inhibitor of apoptosis protein and displays antitumor activity in vivo
}

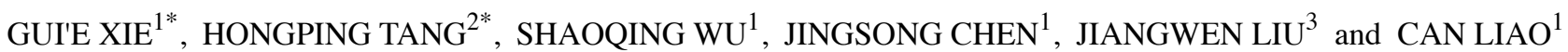 \\ ${ }^{1}$ Guangzhou Women and Children's Medical Center, Guangzhou Medical University, Guangzhou 510623; \\ ${ }^{2}$ Department of Pathology, Shenzhen Maternity and Child Healthcare Hospital Affiliated \\ to Southern Medical University, Shenzhen 518028; ${ }^{3}$ College of Light Industry and \\ Food Science, South China University of Technology, Guangzhou 510640, P.R. China
}

Received March 5, 2014; Accepted May 8, 2014

DOI: 10.3892/ijo.2014.2467

\begin{abstract}
Inhibitors of cyclin-dependent kinases (Cdks) have been reported to have activities in many types of cancer cells by inhibiting $\mathrm{Cdk} 7$ and $\mathrm{Cdk} 9$, which control transcription. SNS-032 is a potent and selective inhibitor of $\mathrm{Cdk} 2, \mathrm{Cdk} 7$ and $\mathrm{Cdk} 9$ and has emerged in clinical trials. Here, we examined the viability of MCF-7 and MDA-MB-435 breast cancer cells in the presence of SNS-032 and observed a dose-dependent inhibition of cellular proliferation in both cell lines. SNS-032 had a direct apoptosis-inducing effect through both the extrinsic and intrinsic apoptotic pathways in breast cancer cells as shown by a dose-dependent increase in Annexin V-positive cells and terminal deoxynucleotidyl transferase-mediated dUTP nick-end labeling (TUNEL)-positive cells, as well as activation of caspase-8, - 9 and poly(ADP-ribose) polymerase (PARP). At the molecular level, SNS-032 induced a marked dephosphorylation of serine 2 and 5 of RNA polymerase (RNA Pol) II and blocked RNA synthesis. Consistent with the inherently rapid turnover rates of their transcripts and proteins, the antiapoptotic proteins Mcl-1 and X-linked inhibitor of apoptosis protein (XIAP) were rapidly reduced on exposure to SNS-032. Our results also indicated that SNS-032 suppressed the growth of breast cancer xenografts in mice. These data demonstrate that the use of SNS-032 may be a rational and novel thera-
\end{abstract}

Correspondence to: Dr Jiangwen Liu, College of Light Industry and Food Science, South China University of Technology, Guangzhou 510640, P.R. China

E-mail: fejwliu@scut.edu.cn

Dr Can Liao, Guangzhou Women and Children's Medical Center, Guangzhou Medical University, Guangzhou 501623, P.R. China

E-mail: liaocan2006@gmail.com

${ }^{*}$ Contributed equally

Key words: cyclin-dependent kinases, SNS-032, apoptosis, Mcl-1, $\mathrm{X}$-linked inhibitor of apoptosis protein, breast cancer peutic strategy for human breast cancer and warrants further clinical investigation.

\section{Introduction}

Breast cancer is the most commonly diagnosed type of cancer and the second leading cause of cancer death in females worldwide (1). Despite the fact that many tumors initially respond to chemotherapy, breast cancer cells can subsequently survive and gain resistance to the treatment $(2,3)$. It is therefore important to identify novel agents with improved pharmacological and toxicological profiles.

Cdks are a group of protein kinases first discovered for their role in regulating cell cycle. Cdks are activated by the formation of a complex with cyclin partners. Specific Cdks operate in distinct phases of the cell cycle $(4,5)$. In addition to their cell cycle regulatory functions, Cdks, especially $\mathrm{Cdk} 7$ and $\mathrm{Cdk} 9$, play important roles in the regulation of RNA Pol II-mediated transcription. The general transcription factor II (TFIIH) complex containing $\mathrm{Cdk} 7$ and cyclin $\mathrm{H}$ first phosphorylates the serine-5 residue of carboxyl-terminal domain (CTD) of the large subunit of RNA Pol II and facilitates the initiation of transcription. Then the Cdk9-cyclin T complex forms the transcription elongation factor $\mathrm{b}(\mathrm{p}-\mathrm{TEFb})$ and phosphorylates the 5,6-dichlorobenzimidazone-1-b-D-ribofuranoside (DRB)sensitive inducing factor and the negative elongation factor, followed by serine- 2 of the CTD to facilitate transcriptional elongation (6-9).

Cdks play a critical role in cancer progression, and misregulation of Cdks is one of the most frequent alterations in human cancer $(4,5,10,11)$. For this reason, Cdks have been considered as very promising therapeutic targets in human malignancies. Over the past decade, the intensive search for pharmacological $\mathrm{Cdk}$ inhibitors has led to several clinical candidates, and the focus on transcriptional Cdks has underlined their antitumor activity (12). Flavopiridol, the first and currently most promising $\mathrm{Cdk}$ inhibitor in preclinical and clinical trials, has demonstrated marked antitumor activity in many types of cancer $(13,14)$. Flavopiridol acts largely through inhibition of Cdk9. When RNA Pol II is repressed after Cdk9 
inhibition, the result is a blockage of transcriptional elongation, which in turn causes decreases in the cellular levels of short-lived proteins including some antiapoptotic molecules such as Mcl-1 and XIAP, and thus promotes the induction of apoptosis $(15,16)$.

SNS-032 is a novel Cdk inhibitor that has emerged in clinical trials. Originally selected as an inhibitor of Cdk2, SNS-032 was later found to be a potent inhibitor of $\mathrm{Cdk} 9$ and Cdk7 (17). SNS-032 is more selective and less cytotoxic compared to flavopiridol, and in vitro studies have shown more potent inhibition of RNA synthesis and cell death induction by SNS-032 than by flavopiridol (18). In this study, we investigated the efficacy of SNS-032 against breast cancer cells. SNS-032 suppressed the proliferation of MCF-7 and MDA-MB-435 breast cancer cells as well as the growth of MDA-MB-435 nude mouse xenografts. Such effects occurred through the suppression of RNA synthesis of antiapoptotic protein Mcl-1 and XIAP, which in turn led to the reduction of the Mcl-1 and XIAP protein and the initiation of apoptosis. Thus, we provide in vitro and in vivo evidence for use of SNS-032 as a promising therapeutic agent for the treatment of breast cancer.

\section{Materials and methods}

Chemicals. SNS-032 was obtained from Selleck Chemicals. LLC (Houston, TX, USA), dissolved in dimethyl sulfoxide (DMSO) to give a stock solution of $10 \mathrm{mM}$ and stored at $-20^{\circ} \mathrm{C}$ in small aliquots.

Cell culture. Human breast cancer cell lines MCF-7 and MDA-MB-435 were cultured as previously described (19).

Cell viability analysis. Cells were seeded in 96-well flat-bottom plates at a density of $1 \times 10^{4}$ cells per well and cultured in a humidified incubator for $24 \mathrm{~h}$, followed by exposure to various concentrations of SNS-032 for additional $48 \mathrm{~h}$. Cell viability was measured by using the MTS (3-(4,5-dimethylthiazol2-yl)-5-(3-carboxymethoxyphenyl)-2-(4-sulfophenyl)-2H-tetrazolium) assay to monitor cell proliferation, according to the manufacturer's recommendations. Briefly, $20 \mu \mathrm{l}$ MTS solution (CellTiter 96Aqueous One Solution reagent, Promega, Madison, WI, USA) was added to each well and incubated for an additional $4 \mathrm{~h}$ at $37^{\circ} \mathrm{C}$. The absorbance was measured on a 96-well plate reader at wavelength $490 \mathrm{~nm}$ (Bio-Tek Synergy 2 , Winooski, VT, USA). Cell growth inhibition was determined using the following formula according to a previously published method: growth inhibition $(\%)=(1-$ OD of treated cells/OD of control cells) x 100\% (19). The half maximal inhibitory concentration $\left(\mathrm{IC}_{50}\right)$ was calculated with Bliss software and the data were analyzed by SPSS. All experiments were performed three times from which mean values were calculated.

Annexin V-FITC (fluorescein isothiocyanate)/propidium iodide (PI) staining assay. Apoptosis was determined by flow cytometry using Annexin V-FITC Apoptosis Detection Kit II (BD Pharmingen, San Diego, CA, USA) according to the manufacturer's instructions. Cells $\left(2 \times 10^{5}\right.$ cells/well $)$ were seeded in $60-\mathrm{mm}$ plates and allowed to settle for $24 \mathrm{~h}$ before treatment with various concentrations $(0,200$ and $400 \mathrm{nM})$ of SNS-032 for an additional $8 \mathrm{~h}$. The cells were harvested by trypsinization, washed twice with cold phosphate-buffered saline (PBS), and then resuspended in $1 \mathrm{X}$ binding buffer at a concentration of $1 \times 10^{6}$ cells $/ \mathrm{ml}$. Following this, $100 \mu \mathrm{l}$ of the sample solution was transferred to a 5-ml culture tube and incubated with $5 \mu \mathrm{l}$ of FITC-conjugated Annexin V and $10 \mu \mathrm{l}$ of PI for $15 \mathrm{~min}$ at room temperature in the dark. Subsequently, $400 \mu \mathrm{l}$ of binding buffer was added to each sample and the samples were analyzed using a flow cytometer (FACSCanto II, Becton-Dickinson, San José, CA, USA).

TUNEL assay. The TUNEL assay for in situ detection of apoptosis was performed by using the DeadEnd ${ }^{\mathrm{TM}}$ Fluorometric TUNEL System assay kit (Promega) according to the manufacturer's instructions. Cells were plated in 24-well flat-bottom plates at a density of $1 \times 10^{5}$ cells per well, treated with $400 \mathrm{nM}$ SNS-032 for $24 \mathrm{~h}$. Following SNS-032 treatment, cells were fixed in $4 \%$ paraformaldehyde at $4^{\circ} \mathrm{C}$ for $25 \mathrm{~min}$. Fixed cells were then permeabilized in $0.1 \%$ Triton X-100 and labeled with fluorescein-12-dUTP using terminal deoxynucleotidyl transferase. After rinsing with PBS, nuclei were counterstained with PI $(1 \mu \mathrm{g} / \mathrm{ml})$ for $15 \mathrm{~min}$. The localized green fluorescence of apoptotic cells was detected by fluorescence microscopy (Zeiss Axiovert 100M, Carl Zeiss, Germany).

Caspase activity assay. Activity of caspase- 8 and -9 was measured using a caspase colorimetric assay kit (Keygen Biotech, China), according to the manufacturer's protocol. Briefly, after treatment of SNS-032 at different concentrations $(0,200$ and $400 \mathrm{nM})$ for $24 \mathrm{~h}$, cells were harvested, washed with PBS and then resuspended in chilled lysis buffer. After incubation on ice for $40 \mathrm{~min}$, cells were centrifuged for $1 \mathrm{~min}$ at $10,000 \mathrm{x}$ g. The supernatant was collected in a fresh tube and protein concentration was determined by the Bradford protein assay kit (Keygen Biotech), according to the manufacturer's protocol. Subsequently, $150 \mu \mathrm{g}$ of each protein sample was diluted with $50 \mu \mathrm{l}$ lysis buffer and added to $50 \mathrm{ml}$ of $2 \mathrm{X}$ reaction buffer containing $10 \mathrm{mM}$ dithiothreitol in a 96 -well plate. Then, $5 \mu$ l of a colorigenic substrate, IETD-pNA (L-isoleucylL-glutamyl-L-Threonyl-L-aspartic-p-nitroanilide acid amide) or LEHD-pNA (L-leucine-L-glutamyl-L-histidyl-L-asparticp-nitroaniline acid amide), was added to each well, and the plate was incubated at $37^{\circ} \mathrm{C}$ in the dark for $4 \mathrm{~h}$. ODs were determined at $405 \mathrm{~nm}$ using a microplate reader (Bio-Tek Synergy 2).

Western blot analysis. After treatment with SNS-032 at different concentrations $(0,200$ and $400 \mathrm{nM})$ for $24 \mathrm{~h}$, cells in each dish, including dead cells floating in medium, were harvested and lysed in 1X sampling buffer. Protein concentrations of the lysates were determined using the bicinchoninic acid protein assay kit (Pierce Biotech, Rockford, IL, USA). An aliquot of the denatured supernatant containing $30 \mu \mathrm{g}$ of protein was subjected to sodium dodecyl sulphate polyacrylamide gel electrophoresis (SDS-PAGE), and then transferred to polyvinylidene fluoride (PVDF) membranes. After blocking with blocking buffer (Tris-buffered saline, i.e. TBS, containing $5 \%$ non-fat milk) for $1 \mathrm{~h}$ at room temperature, the membranes were incubated overnight at $4^{\circ} \mathrm{C}$ with the following specific 
primary antibodies: mouse anti-human caspase- 8 , mouse antihuman caspase-9, rabbit anti-human PARP, rabbit anti-human phospho-RNA Pol II (Ser2), rabbit anti-human phospho-RNA Pol II (Ser5), rabbit anti-human RNA Pol II, rabbit anti-human Cdk7, rabbit anti-human Cdk9, rabbit anti-human Mcl-1, rabbit anti-human XIAP, rabbit anti-human Bcl-2 (Cell Signaling Technology, Beverly, MA, USA); and mouse anti-human GAPDH (ProteinTech, Chicago, IL, USA). Further incubation with appropriate horseradish peroxidase-conjugated secondary antibodies, depending on the primary antibody used, was performed for $1 \mathrm{~h}$ at room temperature. Detection of staining signals was performed by using the enhanced chemiluminescence kit (Thermo Fisher Scientific, Rockford, IL, USA) with Kodak film.

Real-time PCR. Total RNA was extracted using the RNeasy kit (Qiagen, Crawley, UK). Each cDNA template was made from total RNA with reverse transcriptase kit according to the manufacturer's instructions (Invitrogen). Amplification reactions were performed using SYBRW Premix Ex Taq ${ }^{\mathrm{TM}}$ (Takara Shuzo, Kyoto, Japan) in a $25 \mu 1$ volume. The following cycling parameters were used: $30 \mathrm{sec}$ at $95^{\circ} \mathrm{C}$ for initial denaturing, $5 \mathrm{sec}$ at $95^{\circ} \mathrm{C}$ for denaturing and $30 \mathrm{sec}$ at $60^{\circ} \mathrm{C}$ for annealing and extension for the total of 40 cycles. The fold change in mRNA was calculated by the $2^{-\Delta \Delta C t}$ method. All samples were normalized to $18 \mathrm{~S}$ ribosomal RNA, an RNA Pol I transcript that is not modulated by inhibition of RNA Pol II. The primer sequences used were: XIAP-up: 5'-CCATATACCCGAGGAA CCCT-3'; XIAP-dn: 5'-TTTCCACCACAACAAAAGCA-3'; Mcl-1-up: 5'-AAAAGCAAGTGGCAAGAGGA-3'; Mcl-1-dn: 5'-TTAATGAATTCGGCGGGTAA-3'; Bcl-2-up: 5'-AAG ATTGATGGGATCGTTGC-3'; Bcl-2-dn: 5'-TGTGCTTTGCA TTCTTGGAC-3'; 18SrRNA-up:5'-GTAACCCGTTGAACCCC ATT-3'; 18S rRNA-dn: 5'-CCATCCAATCGGTAGTAGCG-3'.

Xenografted tumor model and antitumor effect of SNS-032 in vivo. All animal care and experimental procedures were approved by the Institutional Animal Care and Use Committee of Guangzhou Medical University. Female BALB/c-nu mice $(18-20 \mathrm{~g})$ were purchased from the Experimental Animal Center of Guangzhou University of Chinese Medicine, and were housed in barrier facilities on a 12-h light/dark cycle. On day 0 , human breast cancer MDA-MB- 435 cells $\left(5 \times 10^{6}\right.$ cells in $0.1 \mathrm{ml}$ per mouse) were inoculated subcutaneously in the right mammary gland. On day 6 , the formed tumors were measured, and the mice were randomly divided into a treatment group and a control group. The treatment group received an intraperitoneal (i.p.) dosage of $200 \mu \mathrm{l} \mathrm{SNS}-032$ (15 mg/kg body weight) every 3 days, while animals in the vehicle-control group received i.p. $200 \mu 1$ 0.5\% DMSO solution per mouse. Tumors were measured every 3 days in blinded manner by measuring perpendicular diameters with a digital caliper. The tumor volumes $\left(\mathrm{mm}^{3}\right)$ were calculated using the following formula: volume $=$ width $\mathrm{x}$ width $\mathrm{x}$ length $\mathrm{x} \pi / 6$. Data were presented as the means \pm standard deviation (SD) of six mice in each group. At the end-point of the experiment, all the animals were euthanized, and the tumors were dissected and weighed.

Statistical analysis. The data given in the text are expressed as means $\pm \mathrm{SD}$. Statistical significances of the differences in

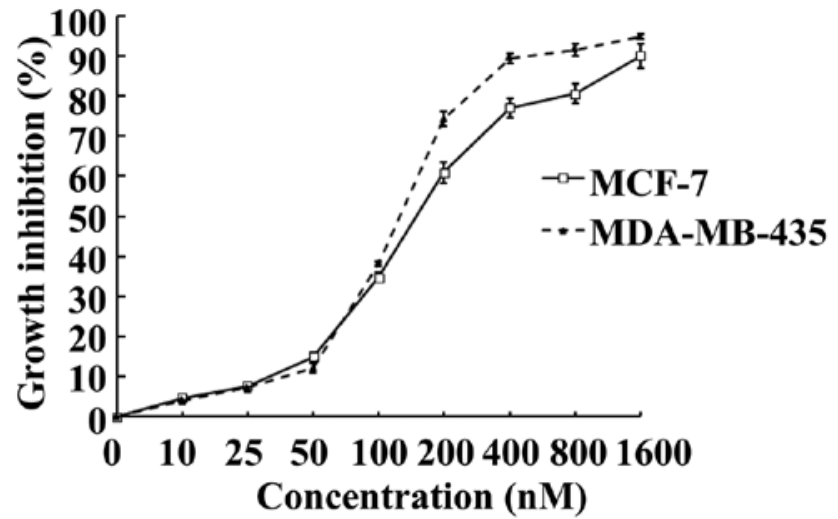

Figure 1. Effect of SNS-032 on growth of breast cancer cell lines MCF-7 and MDA-MB-435. Cells were seeded in 96-well plates and incubated with different concentrations of SNS-032 as noted for $48 \mathrm{~h}$ at $37^{\circ} \mathrm{C}$. Cell viabilities were determined by MTS assay. Data points are presented as means \pm SD of triplicate experiments.

the effects between vehicle-treated mice and SNS-032-treated ones were analyzed by Student's t-test.

\section{Results}

Growth inhibition of human breast cancer cell lines induced by SNS-032. To investigate whether SNS-032 is able to inhibit the growth of human breast cancer cells, its effects on MCF-7 and MDA-MB-435 were examined using MTS assay. Upon treatment of SNS-032 for $48 \mathrm{~h}$, cultured MCF-7 and MDA-MB-435 exhibited markedly inhibited growth, as compared with vehicle-controlled cells in a dose-dependent manner (Fig. 1). Calculated $\mathrm{IC}_{50}$ values, i.e., concentrations of SNS-032 required for decreasing the growth rate of the cells by $50 \%$, were $184.0 \mathrm{nM}$ for MCF-7 and $133.6 \mathrm{nM}$ for MDA-MB-435, respectively.

SNS-032 induces apoptosis in MCF-7 and MDA-MB-435 cells. Apoptosis assays revealed that after treatment of SNS-032 for $8 \mathrm{~h}$ at 200 and $400 \mathrm{nM}$, respectively, numbers of apoptotic MCF-7 and MDA-MB-435 (Annexin $\mathrm{V}^{+} / \mathrm{PI}$ ), as revealed by Annexin- $\mathrm{V}$ binding, markedly increased in a dose-dependent manner (Fig. 2). When the TUNEL assay was performed to assess DNA fragmentation as a late event in the process of apoptosis of MCF-7 and MDA-MB-435 cells, a higher amount of TUNEL-positive cells were visualized in MCF-7 and MDA-MB-435 cells treated for $24 \mathrm{~h}$ with SNS-032 at $400 \mathrm{nM}$, as compared to the control (Fig. 3). The results of two apoptosis assays, i.e., the Annexin V-binding and TUNEL assays, strongly suggested a pro-apoptotic effect of SNS-032 on breast cancer cells.

Activation of both the extrinsic and intrinsic apoptotic pathways by SNS-032. Activation of effector caspases plays a central role in the execution of apoptosis. To further characterize the cell apoptotic process in MCF-7 and MDA-MB-435 cells, pro-apoptotic caspases, i.e., caspase- 9 and -8 , and the effector molecule PARP, were examined on SNS-032 treated or untreated MCF-7 and MDA-MB-435 cells, comparatively. Our results (Fig. 4A) showed that treatments with SNS-032 for 

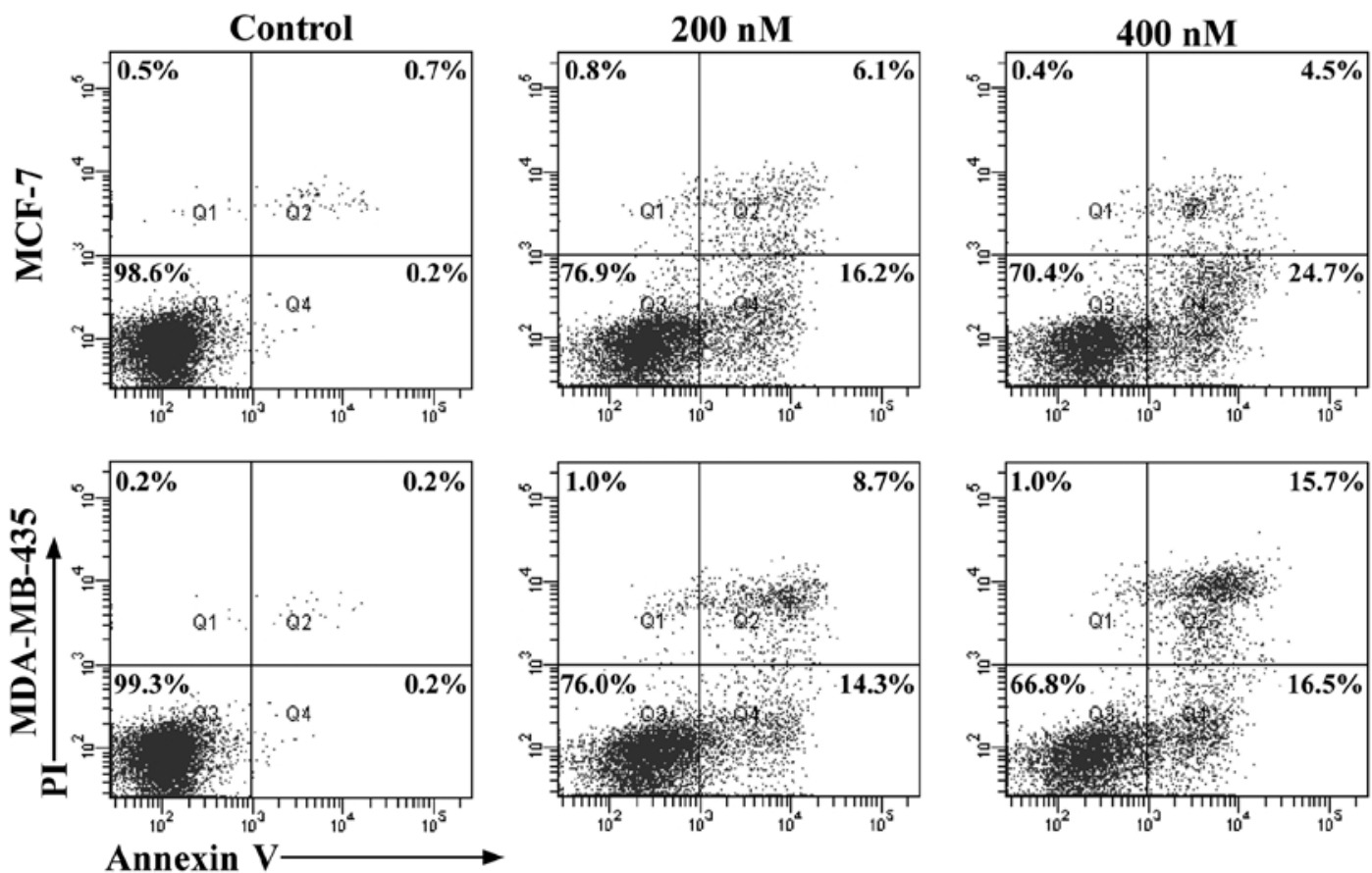

Figure 2. Annexin V-FITC/PI staining of MCF-7 and MDA-MB-435 cells treated with SNS-032. Cells were exposed to different concentrations $(0,200$ and $400 \mathrm{nM}$ ) of SNS-032 for $8 \mathrm{~h}$. Cells were collected and subjected to Annexin V-FITC/PI staining and analyzed by flow cytometry.

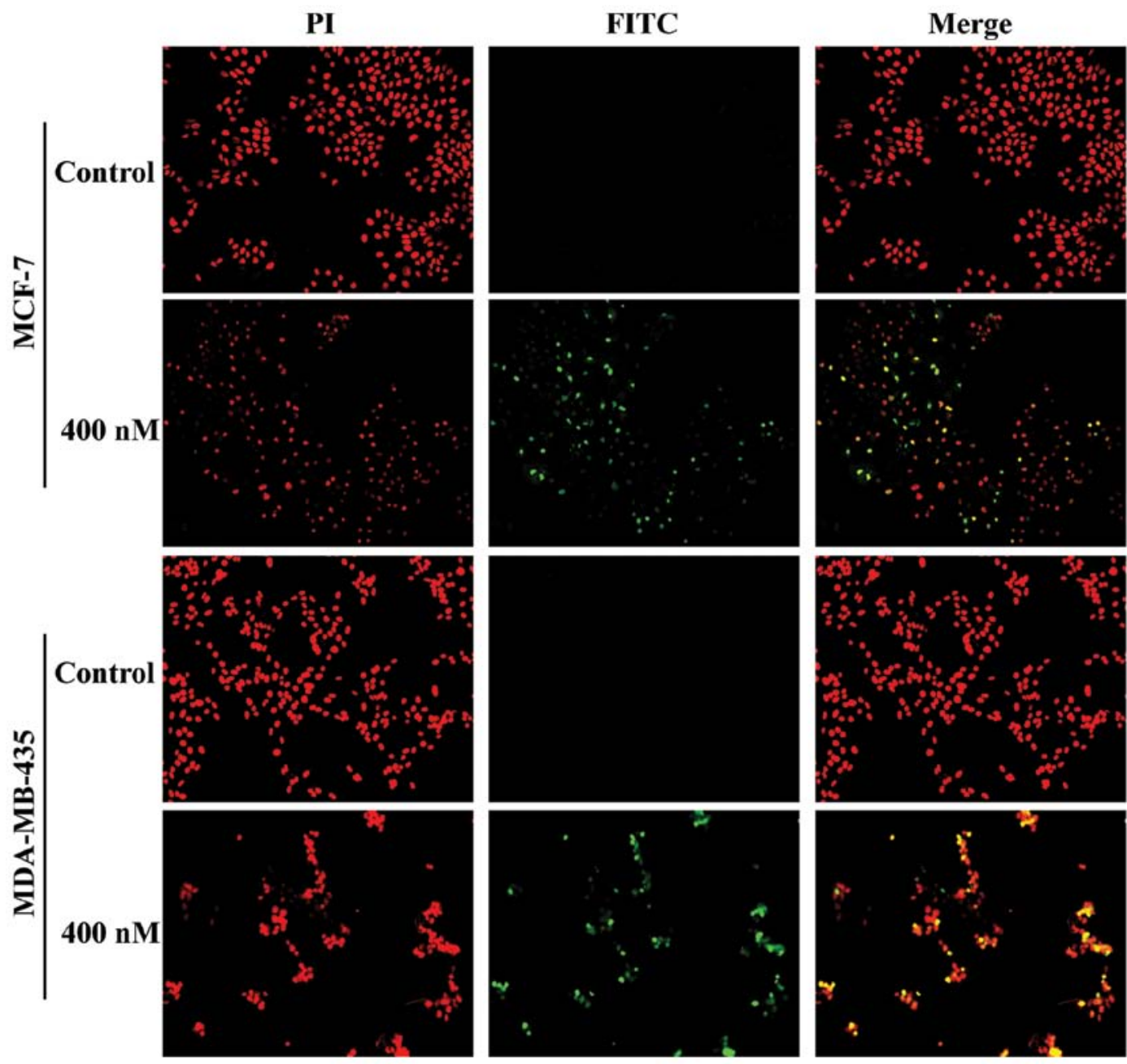

Figure 3. TUNEL assay of MCF-7 and MDA-MB-435 cells treated with SNS-032. Cells were treated with $400 \mathrm{nM}$ SNS-032 as noted for $24 \mathrm{~h}$ and labeled with fluorescein-12-dUTP (green) and PI counterstaining (red). 
A

MCF-7

Control $200 \mathrm{nM} 400 \mathrm{nM}$

Procaspase-8

Cleaved caspase-8

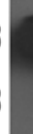

Procaspase-9
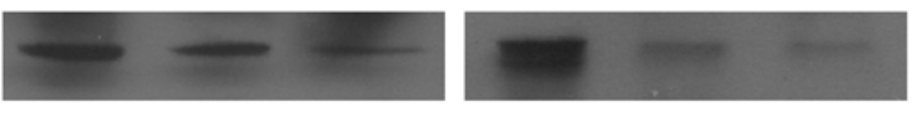

Full-length PARP

Cleaved PARP
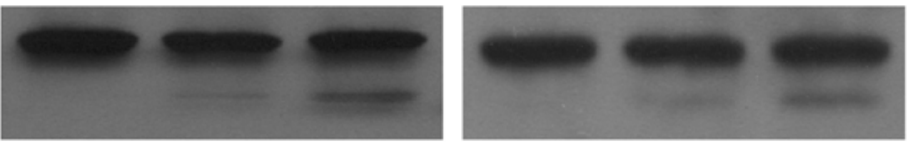

GAPDH
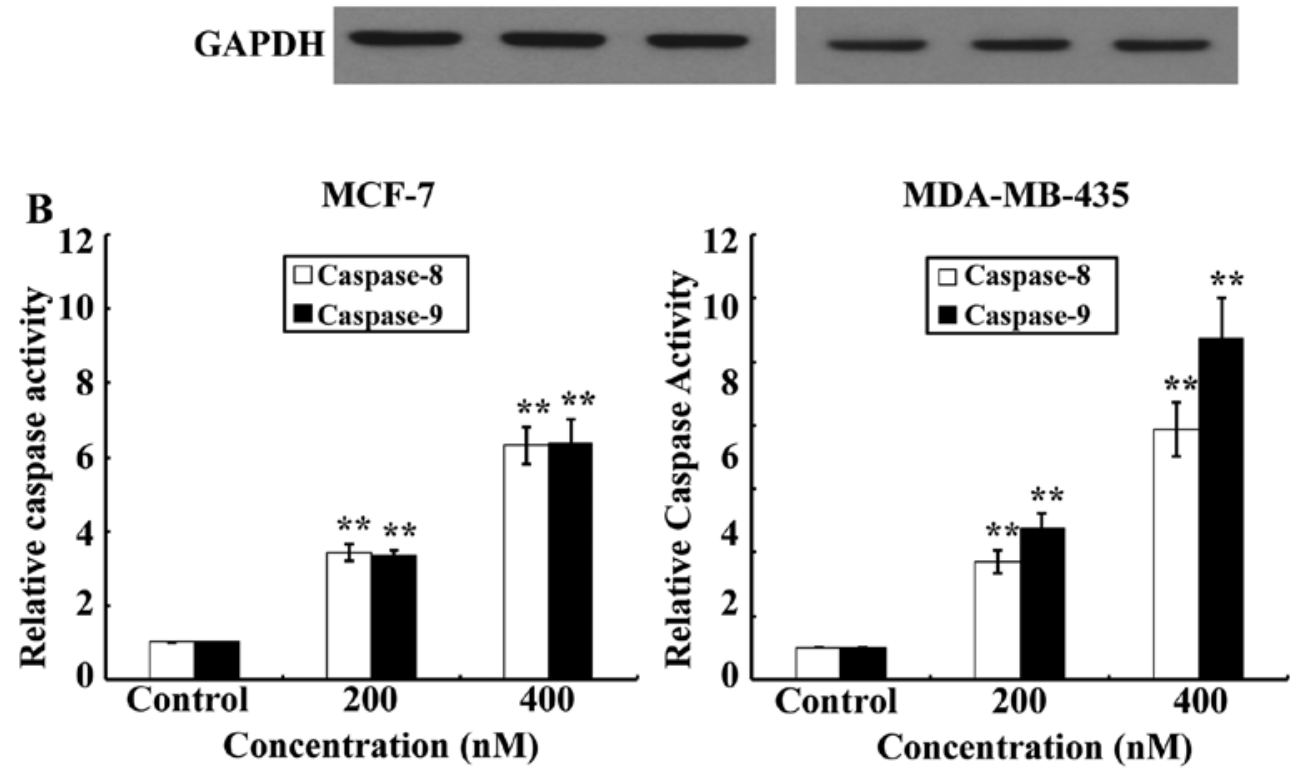

Figure 4. Activation of caspases and PARP in MCF and MDA-MB-435 cells by SNS-032. (A) Western blot analysis of caspases and PARP in MCF and MDA-MB-435 cells after SNS-032 treatment at different concentrations for $48 \mathrm{~h}$ using antibodies against caspase-8, -9 and PARP. GAPDH was used as an internal control. (B) Enzymatic activities of caspases in SNS-032 treated MCF and MDA-MB-435 cells, as assessed by colorimetric assay. The fold-increases in the activities of caspases- 8 and -9 were determined by comparison with those of the vehicle-treated control cells. Results are presented as means \pm SD. ${ }^{* *} \mathrm{p}<0.01$; significant differences compared with the control.

$24 \mathrm{~h}$ dramatically increased activating cleavage of caspase- 8 dose-dependently in MCF-7 and MDA-MB-435 cells. Consistent with the results of western blot analysis, the enzymatic activity of caspase- 8 showed a dose-dependent increase with SNS-032 treatment (Fig. 4B). Concurrently, cleavage of the caspase- 9 precursor and increased caspase- 9 activity were also detected (Fig. 4). Cleavage of PARP from 116 to $85 \mathrm{kDa}$ was clearly demonstrated after SNS-032 treatment in MCF-7 and MDA-MB-435 cells (Fig. 4A). Taken together, these data suggest that apoptosis induced by SNS-032 in breast cancer cells may involve both intrinsic and extrinsic apoptotic pathways.

SNS-032 downregulates antiapoptotic proteins Mcl-1 and XIAP by inhibiting their transcription. Because SNS-032 is a selective inhibitor of transcriptional Cdk7 and 9, consequently disabling RNA Pol II and gene transcription, we further examined its effect on the expression of antiapoptotic proteins Mcl-1, Bcl-2, and XIAP. As shown in Fig. 5, exposing MCF-7 and MDA-MB-435 cells to SNS-032 led to a concentration- dependent decrease in phosphorylated RNA Pol II at Ser2 and Ser 5 but not total protein. The protein levels of Mcl-1 and XIAP were decreased concentration dependently, while there was no significant change in the Bcl-2 protein (Fig. 6A), consistent with a much longer protein half-life $(18,20)$. RT-PCR revealed that SNS-032 decreased the mRNA levels of Mcl-1 and XIAP in MCF-7 and MDA-MB-435 cells (Fig. 6B). These results suggest that the loss of Mcl-1 and XIAP proteins correlates with their transcriptional inhibition due to the blocking of RNA Pol II phosphorylation by SNS-032.

Antitumor effect of SNS-032 on breast cancer xenografts in vivo. To evaluate the antitumor activity of SNS-032 against breast cancer in vivo, we next tested the therapeutic effect of SNS-032 on MDA-MB-435 xenografts in a nude mouse model. When the tumor volumes were assessed on day 6 after inoculation, all the animals in each group were found to have developed spinal cord tumors $(6 / 6$, or $100 \%)$, with a mean volume $( \pm \mathrm{SD})$ of $\sim 30 \mathrm{~mm}^{3}$. The growth of the xenograft tumors were monitored following injection with SNS-032. A 
MCF-7

phospho-RNA pol II (Ser2)

phospho-RNA pol II (Ser5)

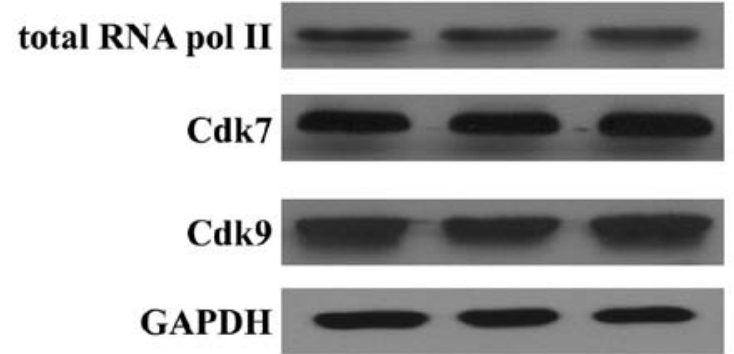

MDA-MB-435
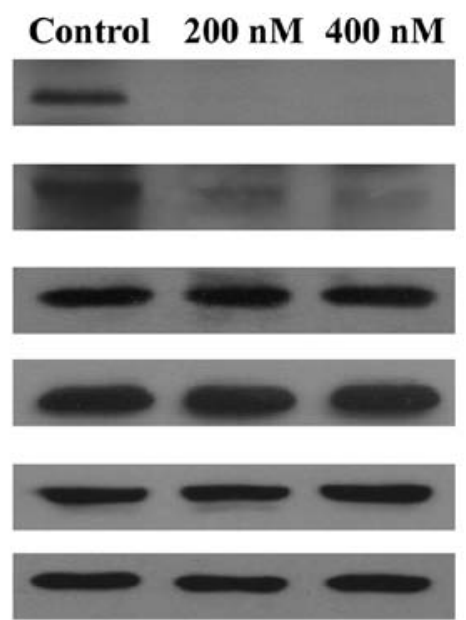

Figure 5. SNS-032 inhibited phosphorylation of RNA Pol II in MCF and MDA-MB-435 cells. Western blot analysis of phospho-RNA Pol II (Ser2 or Ser5), total RNA Pol II, Cdk7 and Cdk9 in MCF and MDA-MB-435 cells after SNS-032 treatment. GAPDH was used as an internal control.

A
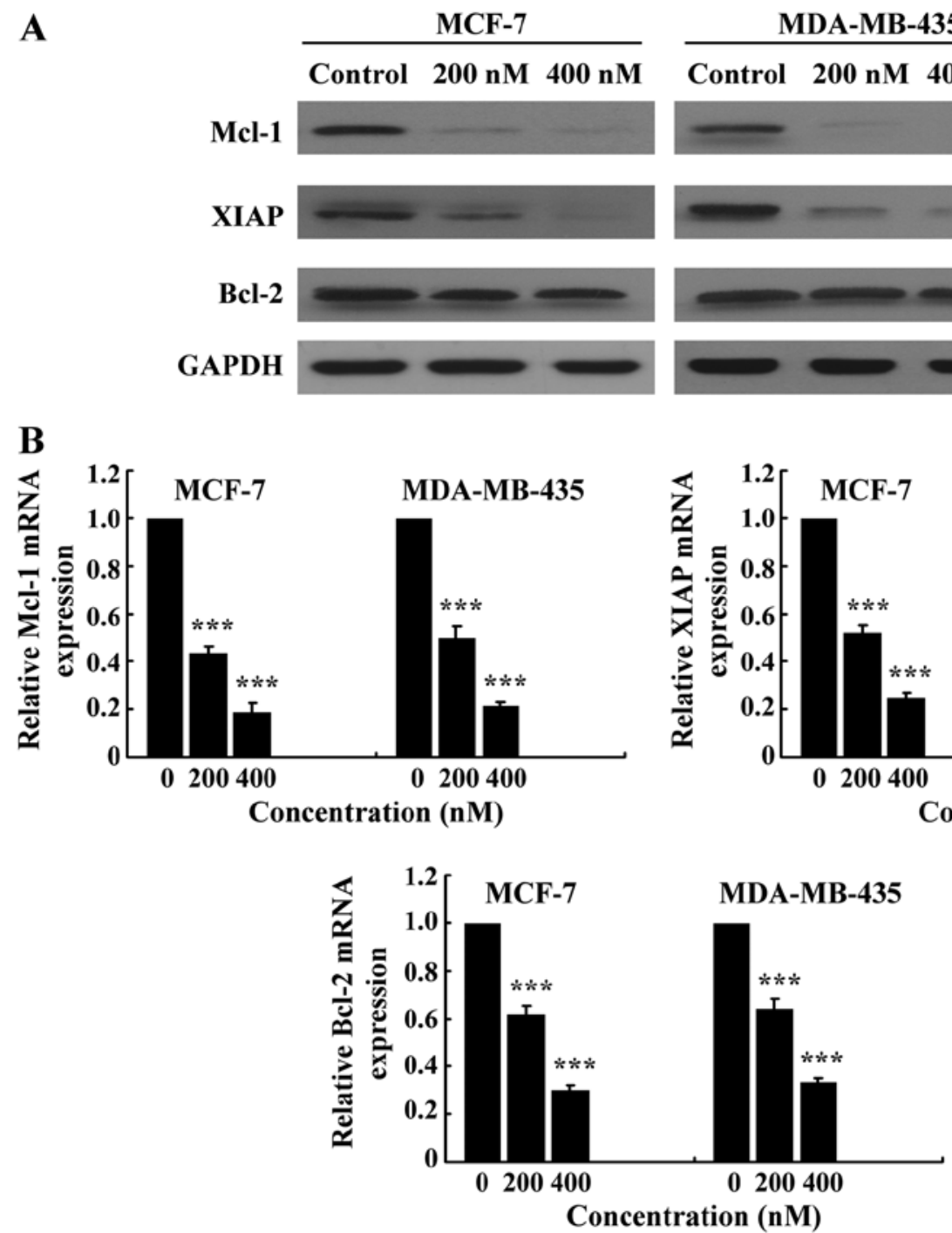

MDA-MB-435
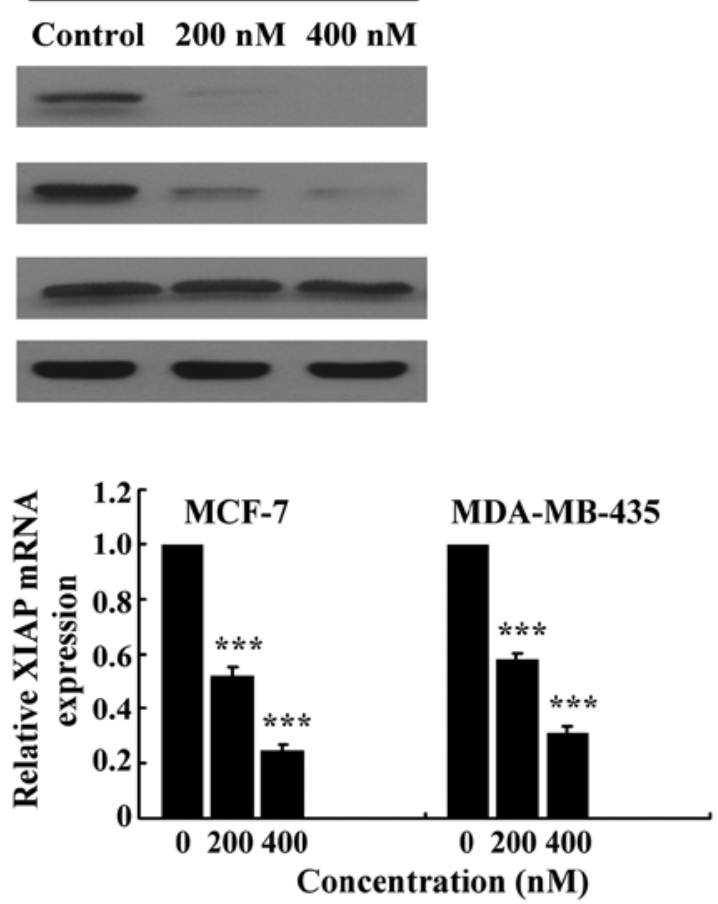

Concentration (nM)

Figure 6. SNS-032 reduced the expression of antiapoptotic proteins by inhibiting transcription. (A) Western blot analysis of Mcl-1, Bcl-2 and XIAP in MCF and MDA-MB-435 cells after SNS-032 treatment. GAPDH was used as an internal control. (B) Real-time PCR quantification of Mcl-1, XIAP and Bcl-2 mRNA expression levels in MCF and MDA-MB-435 cells after SNS-032 treatment. Mcl-1, XIAP and Bcl-2 expression levels are presented as the fold changes relative to those in vehicle-treated control cells and normalized to $18 \mathrm{~S}$ ribosomal RNA. Error bars represent means \pm SD of three independent experiments. **** $\mathrm{p}<0.0001$; significant differences compared with the control. 
A

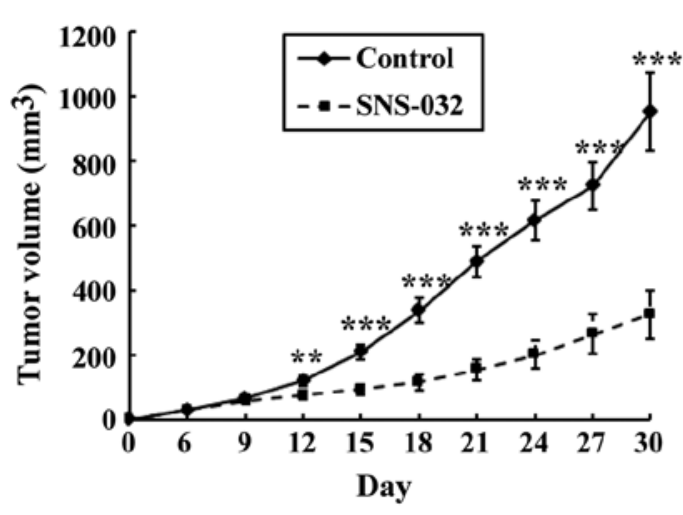

$\mathrm{C}$

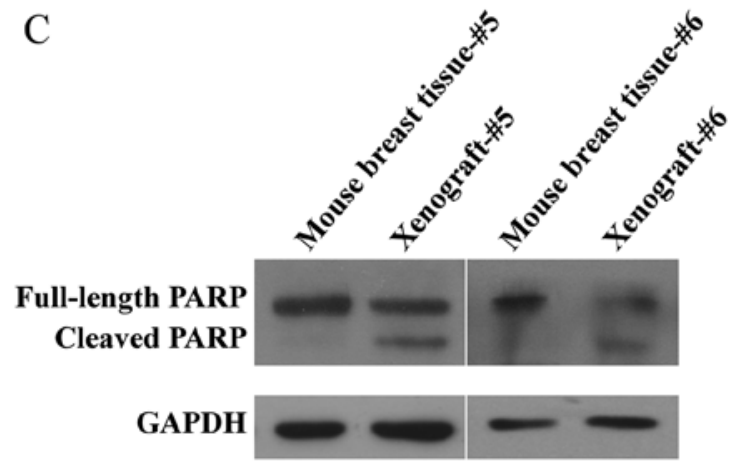

B
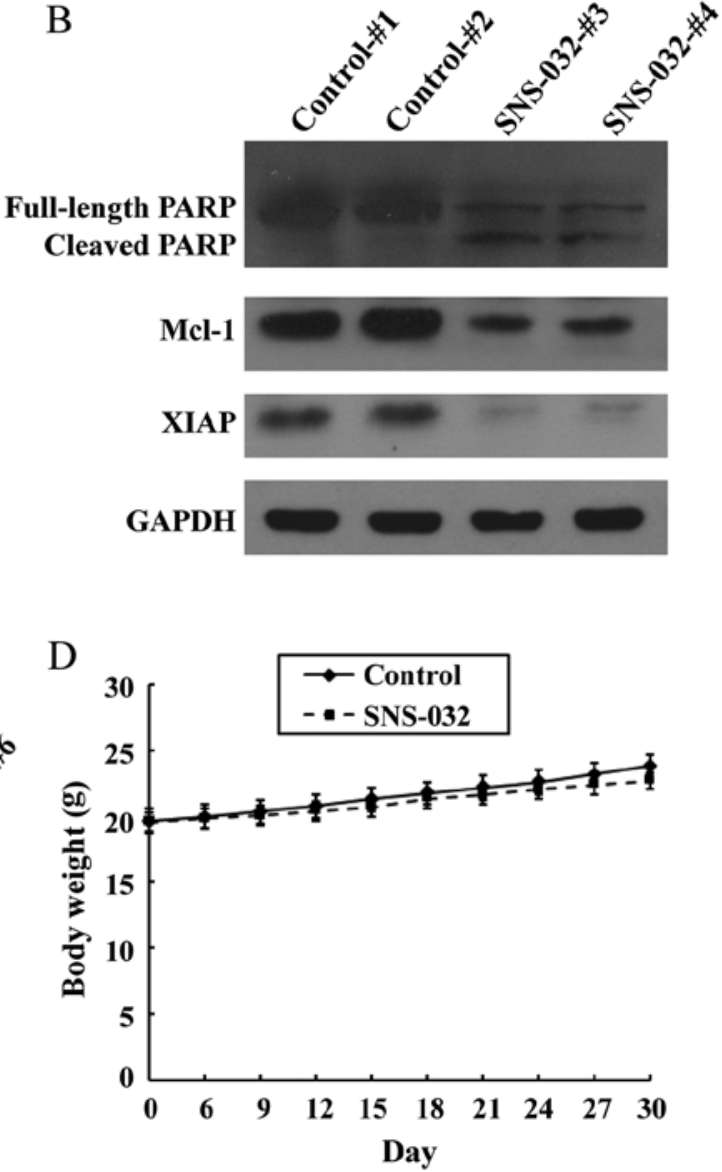

Figuere 7. In vivo antitumor effect of SNS-032 on xenografted MDA-MB-435 tumors in nude mice. Viable MDA-MB-435 cells (5x106 cells/mouse) were inoculated subcutaneously in the right mammary pads of female nude mice. After solid tumor formation, the mice received an i.p. injection of SNS-032 $(15 \mathrm{mg} / \mathrm{kg})$ or vehicle (0.5\% DMSO) every 3 days for approximately one month. (A) Time-response curve of the effect of SNS-032 on the growth of xenografted MDA-MB-435 tumor. (B) Western blot analysis was performed using antibodies against PARP, Mcl-1 and XIAP, with GAPDH used as a loading control, in two representative tumor tissues from mice of each group. (C) Western blot analysis of PARP was performed in two representative tumor tissues from mice treated with SNS-032 and their paired adjacent non-tumor breast tissues. (D) Time-response curve of the effect of SNS-032 on the body weight of the xenografted mice. Results are presented as means \pm SD. ${ }^{* *} \mathrm{p}<0.01,{ }^{* * *} \mathrm{p}<0.0001$; compared with control.

marked inhibition of the growth of the xenografted tumors treated with SNS-032 was observed. After 30 days of drug administration (eight SNS-032 injections), the volume of the xenografted breast tumor was significantly inhibited by $65.77 \%$ in SNS-032-treated nude mice (Fig. 7A).

To assess whether this SNS-032-induced tumor suppression resulted from apoptosis of the grafted cells, western blot analysis of PARP, Mcl-1 and XIAP was carried out. The results showed that injection of SNS-032 induced PARP cleavage in the xenografted breast cancer cells. In contrast, minimal PARP cleavage was detected in tumors excised from vehicle-treated controls. The levels of Mcl-1 and XIAP were significantly lower in tumor tissues from mice treated with SNS-032 than in control treatment (Fig. 7B), so SNS-032 may induce apoptosis and deplete antiapoptotic proteins $\mathrm{Mcl}-1$ and XIAP in vivo.

To assess whether administration of SNS-032 induced apoptosis in normal breast tissues, western blot analysis of PARP was carried out using two representative tumor tissues from mice treated with SNS-032 and their paired adjacent non-tumor breast tissues. The results showed that SNS-032 did not induce apoptosis in normal breast tissues (Fig. 7C).
Meanwhile, no signs of adverse effects, such as discomfort, behavioural changes or weight loss (Fig. 7D), were observed in SNS-032-treated animals. These data strongly suggest that systemically delivered SNS-032 was able to inhibit the growth of established tumors in vivo.

\section{Discussion}

Despite great advances in screening techniques and therapy, breast cancer remains a major health problem worldwide, being the most common cancer and the second leading cause of cancer death among women (1). Typically, the treatment of breast cancer involves antihormonal therapy with the selective estrogen receptor (ER) modulator tamoxifen (21). However, $\sim 30 \%$ of $\mathrm{ER} \alpha(+)$ tumors do not respond to tamoxifen or develop resistance in the course of the treatment $(22,23)$. In addition, the clinical utility of ER modulators is often limited by side effects and is largely ineffective against ER-negative breast cancer $(24,25)$. Therefore, there is an urgent need to explore novel agents that are relatively safe but can suppress growth of both ER-positive and ER-negative human breast cancers. 
Cdks play a critical role in cancer progression making them very promising therapeutic targets in human malignancies. As the development of the pan Cdk inhibitor flavopiridol, more specific Cdk inhibitors have been developed with encouraging results (26-29). In this study, we present our findings on the Cdk inhibitor SNS-032 in breast cancer cells.

SNS-032 is a highly selective and potent inhibitor of Cdks 2, 7 and 9 (17). In addition to its potency and high selectivity, SNS-032 was selected for development based on its favorable characteristics including low protein binding in human serum (18) compared with the high degree of protein binding (92-95\%) seen with flavopiridol (30). Despite promising in vitro activity initially, flavopiridol, the first pan-Cdk inhibitor to enter clinical trials, demonstrated no significant clinical activity in phase I/II studies in patients with solid or hematologic malignancies (31-33). Subsequent investigations revealed significant binding to human plasma proteins that altered free drug level, target cell exposure, and therefore therapeutic activity $(30,34)$. Subsequently, a pharmacokinetically derived schedule of flavopiridol administered as a 30-min intravenous bolus followed by 4 -h continuous intravenous infusion that sustained half maximal inhibitory concentration level was active in refractory chronic lymphocytic leukemia (CLL) (35-37), which indicates high plasma protein binding may be a key reason for the previous lack of clinical activity of flavopiridol. In comparison with flavopiridol, SNS-032 exhibited moderately low protein binding (63\%) in human serum (18). Therefore, SNS-032 has biochemical and pharmacologic properties that differ from flavopiridol, which probably predict differing activities in the clinic.

A phase I trial of SNS-032 in advanced solid tumors including colon cancer, lung cancer, pancreatic cancer and breast cancer showed that this agent was well tolerated in a total of 21 patients enrolled in this study and oral administration may be feasible (38). In another phase I multicenter trial of SNS-032 in patients with advanced B-lymphoid malignancies, including CLL and multiple myeloma (MM), single-agent SNS-032 demonstrated mechanism-based target modulation as well as modest clinical activity in heavily pretreated patients with CLL and MM (39). In this study, we present our findings on SNS-032 in breast cancer cells.

As our data show, SNS-032 displayed potent cytocidal effect on MCF-7 and MDA-MB-435 breast cancer cells, with $\mathrm{IC}_{50}<200 \mathrm{nM}$. SNS-032, at nanomolar concentrations, induced significant apoptosis in MCF-7 and MDA-MB-435 cells as evidenced by activation of caspases, PARP cleavage, Annexin V-positive binding and TUNEL-positive staining. Of the two breast cancer cell lines tested, MCF-7 is relatively well differentiated and estrogen-dependent, whereas MDA-MB-435 is an invasive and estrogen-independent line. The equally, if not more, effective inhibition against MDA-MB-435 cells $\left(\mathrm{IC}_{50}, 133.6 \mathrm{nM}\right)$ by SNS-032 as compared with that against the ER-positive, less invasive and more differentiated MCF-7 cells $\left(\mathrm{IC}_{50}, 184.0 \mathrm{nM}\right)$ warrants further exploration of the possibility to treat ER-negative breast cancer.

Cdk inhibitors that function as transcriptional repressors inhibit RNA Pol II activation by preventing its phosphorylation, the result is a blockage of gene transcription, which in turn causes downregulation of short-lived proteins including some antiapoptotic molecules such as Mcl-1 and XIAP (18).
Mcl-1, an antiapoptotic member of the Bcl-2 family, is among the most frequently amplified genes in human cancer and is essential for the survival of carcinoma cells. Mcl-1 is thought to act by antagonizing pro-apoptotic proteins such as Bim (40). XIAP is a member of the IAP family and plays a key role in cell survival. As the most potent human IAP protein currently identified, XIAP blocks cell death by virtue of inhibition of distinct caspases (41). In this study, our results with breast cancer cells treated with SNS-032 showed a concentrationdependent dephosphorylation of RNA Pol II at serine 5 and 2 . In addition, inhibition of transcription substantially reduced Mcl-1 and XIAP expression, whereas the Bcl-2 protein level remained stable. The rate of decrease in the protein levels of Mcl-1 and XIAP was proportional to their half-lives, with Mcl-1 being the most labile. There was no apparent decrease in Bcl-2 protein level, consistent with a much longer protein half-life $(18,20)$. When Mcl-1 and XIAP are diminished, the balance between the antiapoptotic and proapoptotic proteins will be altered, then irreversibly initiating apoptosis. Previous studies have shown that strategies reducing Mcl-1 or XIAP expression can sensitize breast cancer cells to other chemotherapeutic agents such as lapatinib, etoposide and doxorubicin $(42,43)$. In this regard, our data that exposure of breast cancer cells to SNS-032 decreased Mcl-1 and XIAP expression suggest that SNS-032 might be also effective in enhancing the effects of chemotherapeutics and reducing resistance to conventional chemotherapy in breast cancer.

Consistent with our findings in vitro, SNS-032 significantly suppressed tumor growth in nude mice bearing MDA-MB- 435 tumors, and it was able to induce apoptosis and deplete antiapoptotic proteins Mcl-1 and XIAP in vivo. It should be noted that a smaller effect on PARP cleavage was observed in non-tumor breast tissues. Moreover, no signs of adverse effects, such as discomfort, behavioural changes or weight loss, were observed in SNS-032-treated mice. These results suggest that SNS-032 may be employed as a selective cytotoxic agent for the elimination of cancer cells.

In conclusion, the results of this study demonstrate that SNS-032 has significant antitumor activity against human breast cancer cells both in vitro and in vivo by inducing apoptosis through activation of both extrinsic and intrinsic apoptotic pathways. Dephosphorylation of RNA Pol II and inhibition of Mcl-1 and XIAP RNA synthesis would also contribute to the apoptotic response induced by SNS-032. Given that SNS-032 has favorable characteristics including high Cdk inhibitory selectivity, low protein binding, relatively low toxicity in normal breast cells and significant antitumor activity in human breast cancer cells, it is thought that the use of SNS-032 might be a rational and novel therapeutic strategy for human breast cancer and warrants further clinical investigation.

\section{Acknowledgements}

This study was supported by a grant from the National Natural Science Foundation of China (no. 81101682), a Science and Technology Planning Project of Guangzhou Municipal Health Bureau (no. 201102A213045), a Shenzhen Science and Technology Planning Project (no. 201303072) and a PhD Start-up Fund of Guangzhou Women and Children's Medical Center (no. 201012). 


\section{References}

1. Friedenreich CM: Physical activity and breast cancer: review of the epidemiologic evidence and biologic mechanisms. Recent Results Cancer Res 188: 125-139, 2011.

2. Jafaar ZM, Litchfield LM, Ivanova MM, Radde BN, Al-Rayyan N and Klinge CM: $\beta$-D-glucan inhibits endocrine-resistant breast cancer cell proliferation and alters gene expression. Int J Oncol 44:1365-1375, 2014.

3. Perez EA: Impact, mechanisms, and novel chemotherapy strategies for overcoming resistance to anthracyclines and taxanes in metastatic breast cancer. Breast Cancer Res Treat 114: 195-201, 2009.

4. Gallorini M, Cataldi A and di Giacomo V: Cyclin-dependent kinase modulators and cancer therapy. BioDrugs 26: 377-391, 2012.

5. Canavese M, Santo L and Raje N: Cyclin dependent kinases in cancer: potential for therapeutic intervention. Cancer Biol Ther 13: 451-457, 2012

6. Peterlin BM and Price DH: Controlling the elongation phase of transcription with P-TEFb. Mol Cell 23: 297-305, 2006.

7. Larochelle S, Amat R, Glover-Cutter K, et al: Cyclin-dependent kinase control of the initiation-to-elongation switch of RNA polymerase II. Nat Struct Mol Biol 19: 1108-1115, 2012.

8. Cho SJ, Kim YJ, Surh YJ, Kim BM and Lee SK: Ibulocydine is a novel prodrug Cdk inhibitor that effectively induces apoptosis in hepatocellular carcinoma cells. J Biol Chem 286: 19662-19671, 2011.

9. Wang S and Fischer PM: Cyclin-dependent kinase 9: a key transcriptional regulator and potential drug target in oncology, virology and cardiology. Trends Pharmacol Sci 29: 302-313, 2008.

10. Lapenna S and Giordano A: Cell cycle kinases as therapeutic targets for cancer. Nat Rev Drug Discov 8: 547-566, 2009.

11. Diaz-Padilla I, Siu LL and Duran I: Cyclin-dependent kinase inhibitors as potential targeted anticancer agents. Invest New Drugs 27: 586-594, 2009.

12. Liu X, Shi S, Lam F, Pepper C, Fischer PM and Wang S: CDKI-71, a novel CDK9 inhibitor, is preferentially cytotoxic to cancer cells compared to flavopiridol. Int J Cancer 130: 1216-1226, 2012.

13. Shapiro GI: Preclinical and clinical development of the cyclindependent kinase inhibitor flavopiridol. Clin Cancer Res 10: S4270-S4275, 2004.

14. Wang LM and Ren DM: Flavopiridol, the first cyclin-dependent kinase inhibitor: recent advances in combination chemotherapy. Mini Rev Med Chem 10: 1058-1070, 2012.

15. Gojo I, Zhang B and Fenton RG: The cyclin-dependent kinase inhibitor flavopiridol induces apoptosis in multiple myeloma cells through transcriptional repression and down-regulation of Mcl-1. Clin Cancer Res 8: 3527-3538, 2002.

16. Wittmann S, Bali P, Donapaty S, et al: Flavopiridol down-regulates antiapoptotic proteins and sensitizes human breast cancer cells to epothilone B-induced apoptosis. Cancer Res 63: 93-99, 2003.

17. Conroy A, Stockett DE, Walker D, et al: SNS-032 is a potent and selective CDK 2, 7 and 9 inhibitor that drives target modulation in patient samples. Cancer Chemother Pharmacol 64: 723-732, 2009.

18. Chen R, Wierda WG, Chubb S, et al: Mechanism of action of SNS-032, a novel cyclin-dependent kinase inhibitor, in chronic lymphocytic leukemia. Blood 113: 4637-4645, 2009.

19. Xie G, Zhu X, Li Q, et al: SZ-685C, a marine anthraquinone, is a potent inducer of apoptosis with anticancer activity by suppression of the Akt/FOXO pathway. Br J Pharmacol 159: 689-697, 2010.

20. Blagosklonny MV, Alvarez M, Fojo A and Neckers LM: Bcl-2 protein downregulation is not required for differentiation of multidrug resistant HL60 leukemia cells. Leuk Res 20: 101-107, 1996.

21. Du Y, Shi A, Han B, et al: COX-2 silencing enhances tamoxifen antitumor activity in breast cancer in vivo and in vitro. Int $\mathrm{J}$ Oncol 44: 1385-1393, 2014.

22. Riggins RB, Schrecengost RS, Guerrero MS and Bouton AH: Pathways to tamoxifen resistance. Cancer Lett 256: 1-24, 2007.
23. Zhang B, Zhang $\mathrm{X}$, Tang $\mathrm{B}$, Zheng $\mathrm{P}$ and Zhang $\mathrm{Y}$ : Investigation of elemene-induced reversal of tamoxifen resistance in MCF-7 cells through oestrogen receptor $\alpha(\mathrm{ER} \alpha)$ re-expression. Breast Cancer Res Treat 136: 399-406, 2012

24. Núñez M, Medina V, Cricco G, et al: Glibenclamide inhibits cell growth by inducing G0/G1 arrest in the human breast cancer cell line MDA-MB-231. BMC Pharmacol Toxicol 14: 6, 2013.

25. Jordan VC and Brodie AM: Development and evolution of therapies targeted to the estrogen receptor for the treatment and prevention of breast cancer. Steroids 72: 7-25, 2007.

26. Dickson MA and Schwartz GK: Development of cell-cycle inhibitors for cancer therapy. Curr Oncol 16: 36-43, 2009.

27. Rizzolio F, Tuccinardi T, Caligiuri I, Lucchetti C and Giordano A: CDK inhibitors: from the bench to clinical trials. Curr Drug Targets 11: 279-290, 2010.

28. McInnes C: Progress in the evaluation of CDK inhibitors as antitumor agents. Drug Discov Today 13: 875-881, 2008.

29. Malumbres M and Barbacid M: Cell cycle, CDKs and cancer: a changing paradigm. Nat Rev Cancer 9: 153-166, 2009.

30. Rudek MA, Bauer KS Jr, Lush RM III, et al: Clinical pharmacology of flavopiridol following a 72-hour continuous infusion. Ann Pharmacother 37: 1369-1374, 2003.

31. Byrd JC, Peterson BL, Gabrilove J, et al: Treatment of relapsed chronic lymphocytic leukemia by 72 -hour continuous infusion or 1-hour bolus infusion of flavopiridol: results from Cancer and Leukemia Group B study 19805. Clin Cancer Res 11: 4176-4181, 2005.

32. Flinn IW, Byrd JC, Bartlett N, et al: Flavopiridol administered as a 24-hour continuous infusion in chronic lymphocytic leukemia lacks clinical activity. Leuk Res 29: 1253-1257, 2005.

33. Aklilu M, Kindler HL, Donehower RC, Mani S and Vokes EE Phase II study of flavopiridol in patients with advanced colorectal cancer. Ann Oncol 14: 1270-1273, 2003.

34. Christian BA, Grever MR, Byrd JC and Lin TS: Flavopiridol in the treatment of chronic lymphocytic leukemia. Curr Opin Oncol 19: 573-578, 2007

35. Byrd JC, Lin TS, Dalton JT, et al: Flavopiridol administered using a pharmacologically derived schedule is associated with marked clinical efficacy in refractory, genetically high-risk chronic lymphocytic leukemia. Blood 109: 399-404, 2007.

36. Phelps MA, Lin TS, Johnson AJ, et al: Clinical response and pharmacokinetics from a phase 1 study of an active dosing schedule of flavopiridol in relapsed chronic lymphocytic leukemia. Blood 113: 2637-2645, 2009

37. Lin TS, Ruppert AS, Johnson AJ, et al: Phase II study of flavopiridol in relapsed chronic lymphocytic leukemia demonstrating high response rates in genetically high-risk disease. J Clin Oncol 27: 6012-6018, 2009.

38. Heath EI, Bible K, Martell RE, Adelman DC and Lorusso PM: A phase 1 study of SNS-032 (formerly BMS-387032), a potent inhibitor of cyclin-dependent kinases 2,7 and 9 administered as a single oral dose and weekly infusion in patients with metastatic refractory solid tumors. Invest New Drugs 26: 59-65, 2008.

39. Tong WG, Chen R, Plunkett W, et al: Phase I and pharmacologic study of SNS-032, a potent and selective Cdk2, 7, and 9 inhibitor, in patients with advanced chronic lymphocytic leukemia and multiple myeloma. J Clin Oncol 28: 3015-3022, 2010.

40. Naumann K, Schmich K, Jaeger C, Kratz F and Merfort I: Noxa/ Mcl-1 balance influences the effect of the proteasome inhibitor MG-132 in combination with anticancer agents in pancreatic cancer cell lines. Anticancer Drugs 23: 614-626, 2012.

41. Li QQ, Lee RX, Liang H, Wang G, Li JM, Zhong Y and Reed E: $\beta$-Elemene enhances susceptibility to cisplatin in resistant ovarian carcinoma cells via downregulation of ERCC-1 and XIAP and inactivation of JNK. Int J Oncol 43: 721-728, 2013.

42. Martin AP, Mitchell C, Rahmani M, Nephew KP, Grant S and Dent P: Inhibition of MCL-1 enhances lapatinib toxicity and overcomes lapatinib resistance via BAK-dependent autophagy. Cancer Biol Ther 8: 2084-2096, 2009.

43. Lima RT, Martins LM, Guimarães JE, Sambade C and Vasconcelos MH: Specific downregulation of bcl-2 and xIAP by RNAi enhances the effects of chemotherapeutic agents in MCF-7 human breast cancer cells. Cancer Gene Ther 11: 309-316, 2004. 